\title{
Codevelopment of Healthy and Unhealthy Dietary Behaviors: A Dyadic Examination of Parenting Practices and Adolescent Characteristics
}

\author{
Camilla Piatkowski, $\mathrm{MSc}^{1,2}$; Shelly Keidar, $\mathrm{MPH}^{1,2}$; Nicole Carbert, $\mathrm{MSc}^{1,2}$; \\ Patti-Jean Naylor, $\mathrm{PhD}^{3}$; Heather McKay, $\mathrm{PhD}^{4}$; Rhona Hanning, $\mathrm{PhD}^{5}$; \\ Lucy Le Mare, $\mathrm{PhD}^{6}$; Louise C. Mâsse, $\mathrm{PhD}^{1,2}$
}

\begin{abstract}
Objective: To identify the pathways through which external, parental, and adolescent factors influence adolescents' motivation to adopt healthy dietary behaviors.

Methods: A total of 28 ethnically and socioeconomically diverse (25\% White) families were interviewed in which adolescents (mean age $=12.7$ years; $50 \%$ girls) and parents (mean age $=43.3$ years; $68 \%$ mothers) were interviewed separately. Data were first analyzed thematically, and family summaries, supplemented with diagrams, were then used to identify the processes through which factors influenced adolescents' dietary behaviors.

Results: The analyses identified 3 main motivational pathways (intrinsic motivation, extrinsic motivation, and amotivation), wherein variability and inconsistencies were observed across and within pathways. The unique combination of external factors, parenting profiles, as well as adolescents' willingness to be socialized by family members, differentiated these pathways from each other.

Conclusions and Implications: Health interventions need to target the overall approaches that parents use to socialize their adolescent to make healthy food choices instead of targeting specific parenting practices.

Key Words: parenting practices, dietary behaviors, health promotion, adolescent obesity, obesity prevention (J Nutr Educ Behav. 2021;53:254-260.)

Accepted October 22, 2020. Published online December 10, 2020.
\end{abstract}

Adolescence (13-17 years) is a critical time period to develop and establish healthy dietary behaviors. Within Canada, dietary behaviors begin to deteriorate as children enter

\section{INTRODUCTION}

adolescence during which skipping meals and consuming fast food and sugary drinks, become more prevalent. $^{1,2}$ Thus, it is critical to ascertain factors that influence an adolescent's decision to adopt and maintain healthy dietary behaviors. The

\footnotetext{
${ }^{1}$ BC Children's Hospital Research Institute, Vancouver, British Columbia, Canada

${ }^{2}$ School of Population and Public Health, University of British Columbia, Vancouver, British Columbia, Canada

${ }^{3}$ School of Exercise Science Physical and Health Education, University of Victoria, Victoria, British Columbia, Canada

${ }^{4}$ Faculty of Medicine, Departments of Orthopedics and Family Practice, University of British Columbia, Vancouver, British Columbia, Canada

${ }^{5}$ School of Public Health and Health Systems, University of Waterloo, Waterloo, Ontario, Canada

${ }^{6}$ Faculty of Education, Simon Fraser University, Burnaby, British Columbia, Canada Conflict of Interest Disclosure: The authors have not stated any conflicts of interest.

Address for correspondence: Louise C. Mâsse, PhD, School of Population and Public Health, University of British Columbia, Rm F508, 4480 Oak St, Vancouver, British Columbia V6H 3V4, Canada; E-mail: lmasse@bcchr.ubc.ca

(C) 2020 The Authors. Published by Elsevier Inc. on behalf of Society for Nutrition Education and Behavior. This is an open access article under the CC BY-NC-ND license (http:// creativecommons.org/licenses/by-nc-nd/4.0/)

https://doi.org/10.1016/j.jneb.2020.10.013
}

household environment is a key target of lifestyle behavior interventions, as parents can structure the physical and social environments necessary to support healthy behavior development. $^{3,4}$ Even during adolescence, when peer influence becomes more prominent, parents play an influential role in shaping adolescents' dietary behaviors through food-related parenting practices. $^{5}$ Parents can influence adolescents' diet through the availability of healthy food (eg, fruits and vegetables), modeling of healthy eating behaviors, and active guidance. $^{6}$ By contrast, pressuring strategies were found to be associated negatively with healthy eating. ${ }^{6}$ Thus, parents have the potential to promote and prime their adolescents to adopt and maintain healthy behaviors.

Major gaps still exist within the parenting literature as currently studies (1) examine how parenting practices directly affect adolescents' dietary behaviors, without taking into 
account how the familial context or adolescent factors (eg, knowledge, food preferences, and motivation) can modify these associations; (2) investigate how individual parenting practices influence adolescents' behaviors instead of examining the overall approaches parents use to socialize their adolescent to healthy dietary behaviors; and (3) have not elucidated how sociodemographic factors interact with food parenting practices. ${ }^{6,7}$ Consequently, there is a need to examine how various determinants of healthy eating (eg, health knowledge, goals, motivation, parenting practices, household food availability) interact with one another and whether these interactions significantly affect adolescents' dietary behaviors. Therefore, this study aimed to (1) develop a conceptual framework outlining the factors that influence adolescents' dietary behaviors and (2) identify the pathways through which these factors influence adolescents' motivation to adopt a healthy diet.

\section{METHODS}

\section{Study Participants}

A total of 28 ethnically and socioeconomically diverse families (ie, 1 adolescent and 1 parent) were recruited from 7 public schools in Surrey, British Columbia, Canada (28 parents: 68\% female; 25\% White, 32\% South Asian, 14\% Southeast Asian, 11\% East Asian, 7\% Middle Eastern, and 11\% others; $79 \%$ married; $68 \%$ employed; and $54 \%$ income less than CAD $\$ 60,000$ ( $\sim$ USD \$46,000). Adolescents: 50\% female and all in grade 7 elementary school). The inclusion criteria were (1) the parent was the primary caregiver of the adolescent participant; (2) the adolescent was a grade 7 student; (3) both parent and adolescent were proficient in English; the adolescent had no health condition or disability that limited participation in physical activity, consumption of a normal diet, or required learning assistance; and (4) both parent and adolescent had no history of psychiatric problems or substance abuse.

\section{Data Collection}

The Children's and Women's Research Ethics Board at The University of British Columbia approved the study protocol (H15-01876). The principal investigator and 2 research staffs conducted the semistructured interviews with the adolescent and the parent separately. Interviews were completed from March-May, 2016. The adolescents and the parents were first asked to provide an overview of the adolescent's health behaviors (ie, physical activity, sedentary behavior/screen time, and dietary intake). The participants were then probed to assess whether various aspects of the home and school environments promoted or prevented the adolescent from adopting healthy behaviors. Finally, the influence of parents, peers, and friends was also discussed. The interviews lasted 45-60 minutes and audio-recordings were transcribed verbatim. Each participant received CAD \$35 ( USD \$27).

\section{Data Analysis}

Data analyses in NVivo (version 11, QSR International, Melbourne, Australia, 2015) were guided by grounded theory ${ }^{8}$ and integrated Knafl and Ayres ${ }^{9}$ processes to preserve the family unit. First, a thematic analysis was conducted on the whole dataset to extract the factors that were identified to have potential influence on adolescents' dietary behaviors (focusing on the consumption of fruits, vegetables, processed snacks, processed meals, and sugarsweetened beverages [SSBs], as these were predominantly highlighted in the interviews). After this, Knafl and Ayres $^{9}$ methodologies guided the creation of structured family summaries, using the thematic codes initially identified. The family summaries identified the mechanisms through which external, parental, and adolescent factors interacted with each other. These summaries were supplemented with diagrams to visualize the mechanisms. Finally, the summaries and the diagrams within and across families were compared by constant comparisons to develop a conceptual framework and identify familial subgroups. Two research staff (C.P. and S.K.) and the principal investigator (L.C.M.) developed the thematic codes, the case summaries, and the diagrams. Three family summaries (6 transcripts) were analyzed independently and resulted in further refinement to the analysis. Ten family summaries were coded, summarized, and diagrammed by C.P. and S.K., and the remaining were processed by C.P. and triangulated by S.K. and L.C.M.

\section{RESULTS}

Factors Associated with Adolescents' Dietary Behaviors

Table 1 summarizes the external, parental, and adolescent factors identified in the thematic analyses as having a potential role in shaping adolescents' dietary behaviors. The dyadic analyses of this data, which focused on examining influences within the family units, uncovered how external, parental, and adolescent factors interact to influence adolescents' dietary behaviors, and these processes are summarized in the Figure 1.

\section{Pathways Toward Healthy and Unhealthy Dietary Behaviors}

Figure 2 summarizes the cross-case analyses and highlights the factors that led adolescents to be motivated intrinsically, motivated extrinsically, or unmotivated to carry out healthy dietary behaviors, respectively. Supplementary Tables $1-3$ provide the interim summaries.

Intrinsically motivated adolescents $(n=9)$ generally consumed more fruits and vegetables and limited the intake of processed food, but the consumption of SSBs varied (Figure 2). The parents mentioned the importance of their family living a healthy and active lifestyle, had high levels of health literacy, and had support from other family members (eg, aunts, grandparents). The parents mostly used autonomy supportive and structure practices that were complementary (eg, the adolescent was provided with the opportunity to prepare his/her own lunch; however, parents monitored and redirected as needed). In general, 
Table 1. External, Parental, and Adolescent Factors Identified as Having Potential Influence on Adolescents' Dietary Behaviors

\section{Variables}

Media

Influential people

Social norms

Adolescents'

characteristics

\section{Adolescents'}

motivation

\section{Parental}

characteristics

Health literacy

Socioeconomic status

Contextual factors

Parenting practices Coercive control

Permissiveness

Structure

Autonomy support

\section{Sample Quotes or Definitions}

\section{External factors}

"[My son] likes his Vector [cereal], and it's funny because you know why he gets it? Because there's a hockey player on the front and he thinks it's healthy."

"My aunt is always exercising and she's always eating healthy, so she influences me to eat healthy too."

"She will not bring traditional Indian food [to school] because she'll feel too embarrassed. . .she would prefer a sandwich or a roll."

\section{Adolescent factors}

" can thank my parents for [my healthy diet]."

Some children naturally liked certain food (eg, fruits and vegetables), and others did not.

"Somebody will bring chocolate to school and I usually share with them, but I noticed that isn't healthy and I just stop sharing and I start eating my fruits instead."

"[My parents are] very important. . .because without them I would just be eating junk food all day."

Conscious about own weight or body image

Psychosocial vulnerabilities Severe financial limitations and psychological issues (eg, mental health disorder)

"I know that I need to eat healthy for track [practices]."

"She is the only one who eats [everything] and then she will come to me and say, 'Mom, I'm your healthiest kid, right?'”

"My mom says, 'You should be eating healthy food instead of like junk food' and then I'm just like, 'Whatever.'” Parental factors

Parental knowledge of what constitutes a healthy diet

Modeling a healthy lifestyle and concern about the family's dietary habits

"[My daughter has been asking for] healthier breakfast food but that's more expensive [so] we can't always get that for her."

Marital status, family size, and psychosocial vulnerabilities

"[During] dinner time, we will force him to eat vegetables, meat, or rice."

"There's no set limit when [and] what I can eat or how much I can eat."

"My mom cuts a lot of fruits and veggies for me to eat."

"My mom is teaching me how to cook. So if I'm at home with my sister, I could make something for us to eat, until my parents get home." 


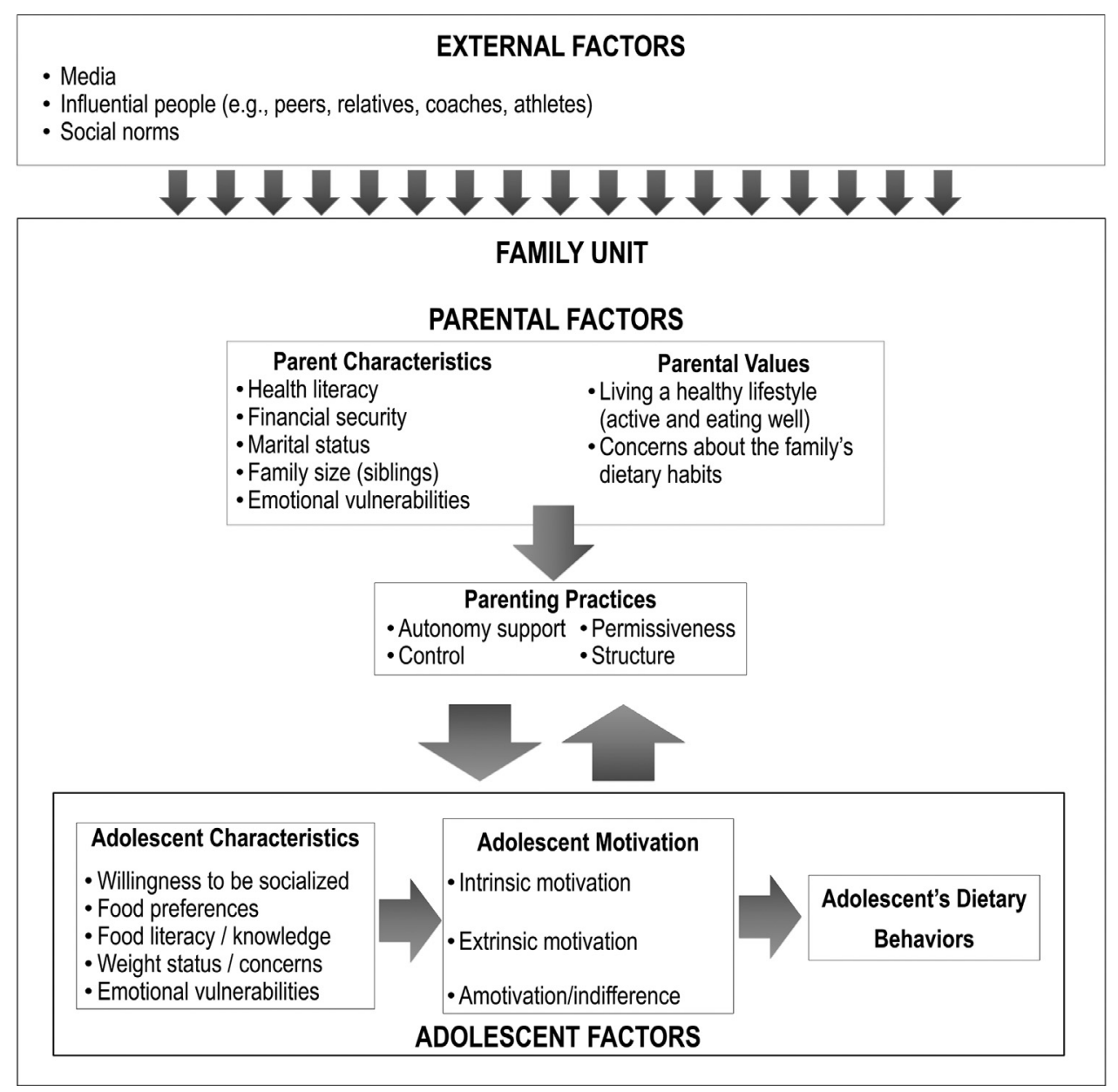

Figure 1. Conceptual model of how external, adolescent, and parental factors influence adolescents' dietary behaviors.

parents were not permissive, and controlling practices were seldom used. Consistency between parents was observed in many families; that is, both parents employed and reinforced the same parenting practices. For adolescent-specific variables, the adolescents expressed their desire to eat a healthy diet or acknowledged household rules related to healthy eating and highlighted the influence of external factors (eg, grandparent or coach) in shaping their values and their willingness to be socialized.

A total of 8 adolescents were motivated extrinsically (eg, pressured or coerced) and demonstrated both healthy (eg, moderate-high intake of fruits and vegetables) and unhealthy dietary behaviors (eg, moderate intake of processed snacks and meals and SSBs) (Figure 2). Parental values regarding healthy living were not mentioned. Rather, parents were concerned that their adolescent consume a healthy/balanced diet, whereas others were more concerned about certain aspects of the adolescent's diet (eg, SSBs intake or daily caloric intake) or wanted their adolescent to learn how to self-regulate their diet without receiving parental support. These parents demonstrated a low-to-moderate level of food literacy, used a variety of practices to control their adolescent's diet (or certain aspects of the diet), many had rules/limits or expectations to limit SSBs intake or increase fruit and vegetable intake, and had parenting practices that were not consistent between mothers and fathers. Control practices were used by a handful of parents (eg, finishing meals and/or consuming enough vegetables). Autonomy supportive practices were seldom used. Finally, structured and situational permissiveness was present within households that facilitated adolescents' exposures to processed meals and snack. When examining the adolescent-specific variables, most were obedient toward their parents. Adolescents felt pressured to practice healthy dietary behaviors as a result of external factors (eg, parental pressures or weight concerns).

Seven adolescents $(n=7)$ were unmotivated or were indifferent to practicing healthy dietary behaviors (Figure 2). Rather, they took part in healthy behaviors only if they were pressured by their parents. All of the adolescents consumed high levels of processed snacks and meals, whereas SSBs and fruit and vegetable intake varied. Parents' intentions (rather than values) were emphasized and reported low health literacy. Most parents were concerned that their adolescent consumed enough 


\begin{tabular}{|c|c|c|c|c|c|}
\hline \multicolumn{3}{|c|}{ Adolescent motivation } & $\begin{array}{l}\text { Intrinsically motivated } \\
\qquad(n=9)\end{array}$ & $\begin{array}{l}\text { Extrinsically motivated } \\
\qquad(n=8)\end{array}$ & \multirow[t]{2}{*}{$\begin{array}{l}\text { Amotivation/Indifference } \\
\qquad(n=7)\end{array}$} \\
\hline \multicolumn{3}{|c|}{ External factors } & & & \\
\hline \multirow{7}{*}{$\begin{array}{l}\text { Adolescent } \\
\text { factors }\end{array}$} & \multicolumn{2}{|c|}{$\begin{array}{l}\text { Willingness to be socialized (i.e. parental food } \\
\text { values are adopted or complied/obeyed with) }\end{array}$} & & & \\
\hline & \multirow{5}{*}{$\begin{array}{l}\text { Early food } \\
\text { preferences for }\end{array}$} & Fruits & & & \\
\hline & & Vegetables & & & \\
\hline & & Processed snacks & & & \\
\hline & & Processed meals & & & \\
\hline & & Sugar-sweetened beverages & & & \\
\hline & \multicolumn{2}{|l|}{ Food literate } & & & \\
\hline \multirow{7}{*}{$\begin{array}{l}\text { Parental } \\
\text { factors }\end{array}$} & \multicolumn{2}{|l|}{ Health literacy } & & & \\
\hline & \multicolumn{2}{|c|}{ Cohesive practices between parents } & & & \\
\hline & \multirow{5}{*}{$\begin{array}{l}\text { Parental } \\
\text { values / } \\
\text { focus for } \\
\text { adolescents' } \\
\text { dietary intake }\end{array}$} & Overall healthy behaviors & & & \\
\hline & & Caloric intake & & & \\
\hline & & Vegetable intake & & & \\
\hline & & Sugar-sweetened beverages & & & \\
\hline & & Other & & & \\
\hline \multirow{4}{*}{$\begin{array}{l}\text { Parenting } \\
\text { practices }\end{array}$} & \multicolumn{2}{|c|}{ Use of coercive control } & & & \\
\hline & \multicolumn{2}{|c|}{ Use of permissive } & & & \\
\hline & \multicolumn{2}{|c|}{ Use of structure } & & & \\
\hline & \multicolumn{2}{|c|}{ Use of autonomy support } & & & \\
\hline \multirow{4}{*}{$\begin{array}{l}\text { Adolescents' } \\
\text { dietary intake }\end{array}$} & \multicolumn{2}{|c|}{ Eat fruits and vegetables } & & & \\
\hline & \multicolumn{2}{|c|}{ Drink sugar-sweetened beverages } & & & \\
\hline & \multicolumn{2}{|c|}{ Eat processed snacks } & & & \\
\hline & \multicolumn{2}{|c|}{ Eat processed meals } & & & \\
\hline
\end{tabular}

High; $\quad$ Moderate; $\quad$ Low; $\square$ Not mentioned;

Not applicable as only 1 parent in the family unit

Figure 2. Overview of how external, adolescent, and parental factors, as well as parenting practices support adolescents to be motivated or unmotivated to adopt healthy dietary behaviors. The shading in each cell represents the percentage of family with each code. The color code for each family was based on evaluating the full transcript for the family and the code assigned to each family was triangulated.

vegetables and/or calories and downplayed the health risk associated with permissive feeding (ie, parents believed that moderate levels of physical activity would compensate for an unhealthy diet). Permissive feeding, along with complementary and negative/unsupportive structure practices (eg, high accessibility to processed food and lack of follow-up action or monitoring from parents) were used by parents. There was limited use of autonomy supportive practices; when employed, they did not result in healthy eating behaviors (eg, allowing the adolescent to prepare his/her own lunches without monitoring or restrictions). Control practices were also used by a few parents. Among the adolescent-specific variables, many preferred processed food over healthier alternatives because of taste preferences. They demonstrated low levels of food literacy. In addition, when presented with the opportunity, adolescents reported overindulging on their preferred food rather than self-regulate their processed food intake.
Four families did not fall under the larger groupings discussed in earlier text. In the transcripts, parents discussed that economic realities and psychological issues disrupted the processes they would like to use to support their adolescent's dietary habits. In addition, the parent-adolescent dynamic can revert, and the adolescent can take on the parent's role with respect to food decisions. Specifically, 1 adolescent was found to predominantly drive the system, as the adolescent highly valued a healthy diet and had a high level of food literacy.

\section{DISCUSSION}

This study uniquely used a dyadic analytical approach to uncover the factors that distinguished whether adolescents were motivated intrinsically, motivated extrinsically, or unmotivated to make healthy food choices. Overall, this study uncovered phenotypes within the family unit that shaped adolescents' selfregulatory processes but (1) variability and inconsistencies existed within each motivational pathway; (2) the profiles of parenting practices, not the use of specific parenting practices, helped differentiate among the different types of motivation; (3) willingness to be socialized or the processes that parents used to influence their adolescent's food identity, as well as external factors, was the most consistent factor associated with each motivational pathway; and (4) for some families, other factors, such as food insecurity or psychological concerns, explained the adolescents' dietary behaviors.

Similar to others, ${ }^{10-13}$ this study found that, in general, positive external role models, adolescents' food preferences, adolescents' willingness to be socialized and adoption of familial values, adolescents' food literacy, healthy familial values, and healthy and cohesive parenting practices (ie, using more autonomy supportive and structuring practices) support adolescents' self-regulatory processes. However, the identified family profiles were not always 
consistent with what others found ${ }^{6}$ nor did they always aligned with selfregulation theory. ${ }^{14}$ Controlling parenting practices has often been associated with poor self-regulatory processes, such as undermining intrinsic motivation and increasing the intake of unhealthy food and decreasing the intake of fruits and vegetables. ${ }^{6,15}$ However, some families that were intrinsically motivated also used coercive approaches. These approaches were seldom used, but when they were, intrinsically motivated adolescents had adopted the healthy food values of their family. These findings raise the question whether it is possible to support healthy regulatory food processes when coercive food parenting practices are used in small doses within an otherwise supportive family unit.

Contrary to expectations, ${ }^{16}$ this study also found that parents used healthy parenting practices (ie, structure and autonomy supportive practice) with unmotivated adolescents. However, a key distinguishing feature was that the parents reported using both healthy and unhealthy parenting practices (ie, structure, autonomy supportive, coercive, and permissive parenting practices). It was unclear whether parents used multiple parenting practices because they were experimenting with them or whether they lacked good parenting skills. In addition, these findings suggest that conflicting parenting practices undermine adolescents' self-regulatory processes, and it appears to lead to amotivation.

This study found that willingness to be socialized distinguished adolescents who were intrinsically motivated, extrinsically motivated, or unmotivated to make healthy food choices. Specifically, adolescents who discussed having food and health values that aligned with their families were more likely to be intrinsically motivated. By contrast, adolescents in the unmotivated group seemed unaware of whether their parents had any values with regard to food. Socializing children to familial values is a process that occurs over time, and it appears that adolescents who were taught the importance of healthy eating when they were young adopt their parents' food intentions. This is consistent with other research ${ }^{17}$ that found familial norms were positively associated with adolescents' fruit and vegetable intake. By establishing certain dietary expectations early on, parents in this study were more likely to teach their adolescent self-regulating skills. Future research might examine feeding practices through a developmental lens to determine factors that support development of lifelong healthy dietary habits.

In addition, cohesive parenting practices supported adolescents' development of healthy food identity and supported their intrinsic motivation to make healthy food choices. By contrast, the adolescents grouped in the extrinsic and unmotivated pathways were more likely to be in a discordant family in which mothers typically reinforced healthy behavior practices, but fathers undermined the mothers' expectations or values. As mothers and fathers have been found to use different childrearing feeding practices, ${ }^{15,18}$ this study highlights the need to align parents' parenting practices to support adolescents' healthy eating.

This study had several limitations. First, poor recall and social desirability bias may have influenced the participants' responses. This bias was partially mitigated by cross-referencing the adolescent and parent transcripts. Second, for many parents English was not their primary language, and language proficiency may have affected how parents conveyed and communicated their experiences, which may have resulted in a loss of details. In addition, immigrant status may have influenced parenting practices and familial perceptions surrounding healthy eating; however, this variable was not collected and could not be analyzed. Third, as volunteers, the families may not be representative of the general population. Fourth, because of its cross-sectional design, this study was unable to capture changes that may have occurred or would have occurred over time. Fifth, the authors acknowledge that external factors (eg, peer influence) increase in adolescence and amplify as adolescents get older, but this aspect was beyond the scope of this article. Finally, owing to the qualitative nature of this study and small sample size, the results cannot be generalized across populations.

This study also had a number of strengths. First, the perspectives of both parent and adolescent were examined and thus, provided a more in-depth evaluation of influences associated with adolescents' diets. Previous studies for the most part relied exclusively on parents' self-reported data. Second, the ethnic and economic diversity of the study participants was well distributed and closely followed Surrey's population in British Columbia; with the exception of White families being underrepresented. ${ }^{19}$ Finally, this is the first study that employed a dyadic perspective to examine the factors that regulate adolescents' motivation to make healthy food choices in a somewhat large qualitative study.

\section{IMPLICATIONS FOR RESEARCH AND PRACTICE}

These findings highlight the need to take a more holistic approach, instead of focusing on specific household factors, to improve the effectiveness of health behavior interventions (eg, consider the profile of parenting practices used in the household by all caregivers). Although parenting practices play a central role in socializing adolescents into adopting healthy eating behaviors, interventions need to tailor their strategies to account for economic realities, psychological issues, and adolescent intrapersonal factors.

\section{ACKNOWLEDGMENTS}

This study was funded by the Canadian Institutes of Health Research, grant no. MOP 340282 and no. MOP 362090. L.C.M. received salary support from the BC Children's Hospital Research Institute. C.P. received University of British Columbia Graduate Support funding and Canadian Institutes of Health Research Frederick Banting and Charles Best Graduate Scholarship award. The authors thank the families who participated in this study for their time and insightful comments. 


\section{SUPPLEMENTARY DATA}

Supplementary data related to this article can be found at https://doi. org/10.1016/j.jneb.2020.10.013.

\section{REFERENCES}

1. Lillico HG, Hammond D, Manske S, Murnaghan D. The prevalence of eating behaviors among Canadian youth using cross-sectional school-based surveys. BMC Public Health. 2014;14:323.

2. Vanderlee L, Manske S, Murnaghan D, Hanning R, Hammond D. Sugar-sweetened beverage consumption among a subset of Canadian youth. J Sch Health. 2014;84:168-176.

3. Lindsay AC, Sussner KM, Kim J, Gortmaker S. The role of parents in preventing childhood obesity. Future Child. 2006;16:169-186.

4. Ritchie LD, Welk G, Styne D, Gerstein DE, Crawford PB. Family environment and pediatric overweight: what is a parent to do. J Am Diet Assoc. 2005;105(5 suppl 1):S70-S79.

5. Pedersen S, Grønhøj A, Thøgersen J. Following family or friends. Social norms in adolescent healthy eating. Appetite. 2015;86:54-60.

6. Yee AZH, Lwin MO, Ho SS. The influence of parental practices on child promotive and preventive food consumption behaviors: a systematic review and meta-analysis. Int J Behav Nutr Phys Act. 2017;14:47.

7. Blaine RE, Kachurak A, Davison KK, Klabunde R, Fisher JO. Food parenting and child snacking: a systematic review. Int J Behav Nutr Phys Act. 2017;14:146.

8. Corbin JM, Strauss A. Grounded theory research: procedures, canons, and evaluative criteria. Qual Sociol. 1990; 13:3-21.

9. Knafl KA, Ayres L. Managing large qualitative data sets in family research. J Fam Nurs. 1996;2:350-364.

10. Backman DR, Haddad EH, Lee JW, Johnston PK, Hodgkin GE. Psychosocial predictors of healthful dietary behavior in adolescents. J Nutr Educ Behav. 2002;34:184-192.

11. Burgermaster M, Contento I, Koch P, Mamykina L. Behavior change is not one size fits all: psychosocial phenotypes of childhood obesity prevention intervention participants. Transl Behav Med. 2018;8:799-807.

12. O'Dea JA. Why do kids eat healthful food? Perceived benefits of and barriers to healthful eating and physical activity among children and adolescents. J Am Diet Assoc. 2003;103: 497-501.
13. Kalavana TV, Maes S, De Gucht V. Interpersonal and self-regulation determinants of healthy and unhealthy eating behavior in adolescents. $J$ Health Psychol. 2010;15:44-52.

14. Bandura A. Social cognitive theory of self-regulation. Organ Behav Hum Decis Process. 1991;50:248-287.

15. Loth KA, MacLehose RF, Fulkerson JA, Crow S, Neumark-Sztainer D. Foodrelated parenting practices and adolescent weight status: a population-based study. Pediatrics. 2013;131:e1443-e1450.

16. Deci EL, Ryan RM. Facilitating optimal motivation and psychological wellbeing across life's domains. Can Psychol. 2008;49:14-23.

17. Granner ML, Evans AE. Measurement properties of psychosocial and environmental measures associated with fruit and vegetable intake among middle school adolescents. J Nutr Educ Behav. 2012;44:2-11.

18. Lloyd AB, Lubans DR, Plotnikoff RC, Collins CE, Morgan PJ. Maternal and paternal parenting practices and their influence on children's adiposity, screen-time, diet and physical activity. Appetite. 2014;79:149-157.

19. Statistics Canada. Census profile, 2016 census. https://www12.statcan.gc.ca/ census-recensement/2016/dp-pd/prof. Accessed November 16, 2020. 